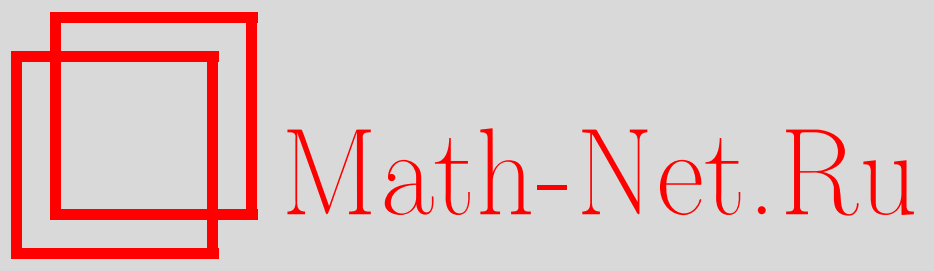

И. О. Глебов, В. В. Ерёмин, Экспоненциально затухающие операторы для гармонического осциллятора, линейно связанного с термостатом, ТМФ, 2010, том 162, номер 2, 243-253

DOI: https://doi.org/10.4213/tmf6467

Использование Общероссийского математического портала Math-Net.Ru подразумевает, что вы прочитали и согласны с пользовательским соглашением http://www . mathnet.ru/rus/agreement

Параметры загрузки:

IP : 3.82 .47 .9

26 апреля 2023 г., 15:25:33

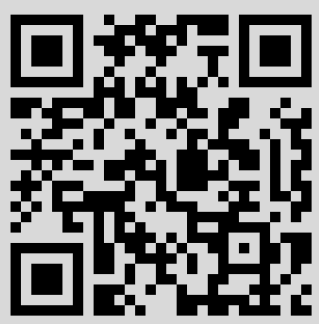




\title{
ЭКСПОНЕНЦИАЛЬНО ЗАТУХАЮЩИЕ ОПЕРАТОРЫ ДЛЯ ГАРМОНИЧЕСКОГО ОСЦИЛЛЯТОРА, ЛИНЕЙНО СВЯЗАННОГО С ТЕРМОСТАТОМ
}

\begin{abstract}
Рассмотрена задача о диссипативной динамике гармонического осциллятора, линейно связанного с термостатом. Показано, что, кроме средней энергии, существует бесконечный ряд экспоненциально затухающих по времени величин, которые являются средними значениями полиномов гамильтониана системы. Получен спектр соответствующих времен релаксации. Предложена методика представления временнь́х характеристик системы через операторы, соответствующие экспоненциально убывающим наблюдаемым. Получена рекуррентная формула для этих операторов.
\end{abstract}

Ключевые слова: квантовая теория диссипации, теория Редфилда, редуцированная матрица плотности, матрица корреляционных функций, гармонический осциллятор.

\section{1. ВВЕДЕНИЕ}

Теория Редфилда [1] широко используется для описания различных диссипативных процессов на квантовом уровне. Так, учет перехода от когерентного движения к равновесному распределению важен для описания сверхбыстрых биохимических процессов, происходящих за времена порядка сотен фемтосекунд [2]-[6].

В ряде теоретических работ был проведен численный анализ колебательной динамики в многоуровневых системах с диссипацией [7]-[9] и показана применимость теории Редфилда для моделирования неадиабатических переходов между электронными состояниями реагентов и продуктов химических реакций. Использование подобных подходов помогает проследить закономерности влияния молекулярных параметров и условий проведения на динамику биохимических реакций (например, переноса электрона в реакционном центре фотосинтеза [10]) и дает хорошее согласие с экспериментальными данными [11].

Однако большая часть работ, посвященных описанию диссипативной динамики, основана на численных расчетах. В то же время аналитические подходы, когда они возможны, позволяют получить четкую физическую картину рассматриваемых

* Московский государственный университет, Москва, Россия.

E-mail: glebov_io@mail.ru,vv_er@mail.ru 
явлений и охарактеризовать зависимость поведения системы от параметров используемых моделей. Данная статья является продолжением предыдущих работ, направленных на аналитическое описание диссипативной динамики в гармоническом приближении [12]. В работе [13] были получены замкнутые выражения для элементов тензора Редфилда, что позволило найти в явном виде зависимость средней колебательной энергии от времени. В настоящей работе мы обобщаем этот результат на целый класс операторов, для которых обнаружено экспоненциальное затухание средних значений по времени и найдены соответствующие времена релаксации.

Предлагается процедура построения этого класса операторов через тепловое расщепление операторов рождения и уничтожения и показывается, как с их помощью можно определить временны́е характеристики осциллятора, включая заселенности отдельных колебательных уровней, не решая в явном виде уравнения Редфилда.

\section{2. МОДЕЛЬ}

Рассмотрим задачу о релаксации одномерного гармонического осциллятора, линейно связанного с термостатом. Полный гамильтониан может быть представлен как сумма слагаемых, соответствующих наблюдаемой системе $H_{\mathrm{S}}$, термостату $H_{\mathrm{B}}$ и их взаимодействию $V$. Оператор взаимодействия линеен по координатам как осциллятора, так и термостата [13]:

$$
\begin{gathered}
\mathbf{H}=\mathbf{H}_{\mathrm{S}}+\mathbf{H}_{\mathrm{B}}+\mathbf{V}, \\
\mathbf{H}_{\mathrm{S}}=\hbar \omega\left(b^{+} b+\frac{1}{2}\right), \quad \mathbf{H}_{\mathrm{B}}=\sum_{\mu} \hbar \omega_{\mu}\left(a_{\mu}^{+} a_{\mu}+\frac{1}{2}\right), \\
\mathbf{V}=\hbar \sum_{q} g_{q}\left(b^{+} a_{q}+b a_{q}^{+}\right),
\end{gathered}
$$

где $\omega$ и $\omega_{\mu}$ - частота осциллятора и $\mu$-й колебательной моды термостата соответственно, $b^{+}$и $b$ - операторы рождения и уничтожения для гармонического осциллятора, $a_{\mu}^{+}$и $a_{\mu}-$ для термостата.

В рамках теории Редфилда [1] динамика редуцированной матрицы плотности, соответствующей наблюдаемой системе, описывается системой дифференциальных уравнений для ее элементов [14]:

$$
\frac{d}{d t} \sigma_{\mu \nu}(t)=-i \omega_{\mu \nu} \sigma_{\mu \nu}(t)+\sum_{\kappa, \lambda} R_{\mu \nu \kappa \lambda} \sigma_{\kappa \lambda}(t),
$$

где $\omega_{\mu \nu}=\left(E_{\mu}-E_{\nu}\right) / \hbar$ - частота перехода между уровнями $\mu$ и $\nu, R_{\mu \nu \kappa \lambda}-$ элементы тензора релаксации. Эти уравнения для нашей задачи имеют существенно более простой вид [13]:

$$
\begin{aligned}
\frac{d}{d t} \sigma_{j k}(t)= & -\left(i \omega_{j k}+\frac{1}{2} J(\omega)[n(\omega)(j+k+2)+(1+n(\omega))(j+k)]\right) \sigma_{j k}(t)+ \\
& +J(\omega) n(\omega) \sqrt{j k} \sigma_{j-1, k-1}(t)+ \\
& +J(\omega)(1+n(\omega)) \sqrt{(j+1)(k+1)} \sigma_{j+1, k+1}(t)
\end{aligned}
$$


для произвольных элементов и

$$
\begin{array}{rl}
\frac{\partial}{\partial t} \sigma_{i i}(t)=i & J(\omega) n(\omega)\left[\sigma_{i-1, i-1}(t)-\frac{1+n(\omega)}{n(\omega)} \sigma_{i i}(t)\right]- \\
& -(i+1) J(\omega) n(\omega)\left[\sigma_{i i}(t)-\frac{1+n(\omega)}{n(\omega)} \sigma_{i+1, i+1}(t)\right]
\end{array}
$$

для диагональных элементов, где $n(\omega)$ - среднее равновесное колебательное квантовое число при температуре $T$ :

$$
n(\omega, T)=\frac{1}{e^{\hbar \omega /(k T)}-1}
$$

а $J(\omega)$ - спектральная функция термостата:

$$
J(\omega)=2 \pi \sum_{q} g_{q}^{2} \delta\left(\omega-\omega_{q}\right),
$$

которая в случае большого количества колебательных мод термостата может считаться непрерывной [15]. Далее мы рассмотрим уравнение (4) для диагональных членов матрицы плотности, которые представляют собой заселенности отдельных колебательных уровней.

Система уравнений (4) является линейной, поэтому ее решение можно записать в виде

$$
\sigma_{i i}(t)=\sigma_{i i}(\infty)+\sum_{j} c_{i j} e^{-k_{j} t}
$$

где все $k_{j}$ - некоторые положительные числа. Это следует из действительности диагональных элементов матрицы плотности и существования предельных значений $\sigma_{i i}(\infty)[13]$. Выполнив линейное преобразование диагональных элементов $\sigma_{i i}(t)$, для каждого числа $k_{j}$ можно перейти к одноэкспоненциальной зависимости:

$$
\sum_{i} d_{i j} \sigma_{i i}(t)=C e^{-k_{j} t}
$$

Далее мы покажем, как, не решая систему уравнений (4), можно получить достаточную информацию о диссипативной динамике рассматриваемой системы.

Любую линейную комбинацию диагональных элементов матрицы плотности можно представить как среднее значение некоторого оператора, коммутирующего с гамильтонианом системы:

$$
\begin{gathered}
\left\langle\mathbf{D}_{j}\right\rangle(t)=\sum_{i} d_{i j} \sigma_{i i}(t)=\operatorname{tr}\left(\mathbf{D}_{j} \cdot \boldsymbol{\sigma}\right), \\
\mathbf{D}_{j}=\sum_{i} d_{i j}|i\rangle\langle i|,
\end{gathered}
$$

где $|i\rangle$ - собственные векторы гамильтониана системы. Следовательно, задача нахождения функций вида (7) может быть переформулирована как задача поиска 
операторов, релаксация средних значений которых имеет одноэкспоненциальный характер:

$$
\begin{aligned}
\left\langle\mathbf{D}_{j}\right\rangle(t) & =e^{-k_{j} t}\left\langle\mathbf{D}_{j}\right\rangle(0), \\
\frac{d}{d t}\left\langle\mathbf{D}_{j}\right\rangle & =-k_{j}\left\langle\mathbf{D}_{j}\right\rangle
\end{aligned}
$$

Два оператора, удовлетворяющие условию (10), известны - это единичный оператор (нормировка матрицы плотности), соответствующий $k_{0}=0$, и гамильтониан системы за вычетом равновесной энергии $\left(k_{1}=J(\omega)\right)[13]$ :

$$
\begin{gathered}
\operatorname{tr}(\boldsymbol{\sigma})=1, \\
\left\langle\mathbf{H}_{\mathrm{S}}-E_{\text {equil }}\right\rangle=E(t)-E_{\text {equil }}=\left[E_{0}-E_{\text {equil }}\right] e^{-J(\omega) t} .
\end{gathered}
$$

Рассмотрим $n$-ю степень энергии. Производная по времени среднего значения $n$-й степени гамильтониана является линейной комбинацией всех предшествующих степеней гамильтониана (см. приложение А):

$$
\frac{\partial}{\partial t}\left\langle\frac{E^{n}}{\hbar^{n} \omega^{n}}\right\rangle(t)=-n J(\omega)\left\langle\frac{E^{n}}{\hbar^{n} \omega^{n}}\right\rangle(t)+\sum_{j=1}^{n} \alpha_{n j}\left\langle\frac{E^{n-j}}{\hbar^{n-j} \omega^{n-j}}\right\rangle(t),
$$

где $\alpha_{n j}$ - некоторые числа, зависящие от температуры.

Из уравнения (12) следует, что спектр констант затухания $\left\langle E^{n}\right\rangle(t)$ может быть получен из спектра $\left\langle E^{n-1}\right\rangle(t)$ добавлением одного числа, а именно $k_{n}=n J(\omega)$. С учетом значений $k_{0}$ и $k_{1}$ по индукции легко доказать, что спектр констант затухания содержит все числа вида $n J(\omega)$, а времена релаксации имеют вид

$$
\tau_{n}=\frac{1}{k_{n}}=\frac{1}{n J(\omega)}=\frac{\tau_{1}}{n} .
$$

Опишем процедуру построения операторов (9), соответствующих полученным константам. Эти операторы являются полиномами относительно гамильтониана. Однако их коэффициенты выражаются через рекуррентные формулы с температурной зависимостью, что затрудняет дальнейшие выкладки. В связи с этим общий вид операторов (9) лучше искать через операторы, не зависящие от температуры. Определим один из таких наборов, воспользовавшись процедурой диагонализации матрицы корреляционных функций [12].

Оператор взаимодействия $\mathbf{V}$ в представлении взаимодействия имеет вид

$$
\mathbf{V}_{I}(t)=e^{-i\left(\mathbf{H}_{\mathrm{S}}+\mathbf{H}_{\mathrm{B}}\right) t / \hbar} \mathbf{V} e^{i\left(\mathbf{H}_{\mathrm{S}}+\mathbf{H}_{\mathrm{B}}\right) t / \hbar}=e^{-i \omega t} b^{+} \sum_{j} g_{j} a_{j, I}(t)+e^{i \omega t} b \sum_{j} g_{j} a_{j, I}^{+}(t),
$$

следовательно, мы имеем двумерное пространство [12] операторов системы и две частоты перехода $\omega$ и $-\omega$. Операторы термостата, соответствующие им, имеют вид

$$
\begin{gathered}
\mathbf{Q}(\tau)=\sum_{j} g_{j} a_{j} e^{i \omega_{j} \tau}, \\
\mathbf{Q}^{+}(\tau)=\sum_{j} g_{j} a_{j}^{+} e^{-i \omega_{j} \tau} .
\end{gathered}
$$


После ортогонализации операторов термостата и выбора резонансных слагаемых [12] оператор энергии взаимодействия перепишется следующим образом (см. приложение Б):

$$
\mathbf{V}=g_{\omega}\left(q^{+}(\omega) b \sqrt{n(\omega, T)}+q(\omega) b^{+} \sqrt{1+n(\omega, T)}\right)+\mathbf{V}^{\prime}
$$

где

$$
\begin{gathered}
q\left(\omega_{-\mu}\right)=a_{\mu}^{+} \sqrt{\frac{1-e^{-\hbar \omega_{\mu} /(k T)}}{e^{-\hbar \omega_{\mu} /(k T)}}}=a_{\mu}^{+}\left[n\left(\omega_{\mu}, T\right)\right]^{-1 / 2} \\
q\left(\omega_{\mu}\right)=a_{\mu} \sqrt{\frac{1}{1-e^{-\hbar \omega_{\mu} /(k T)}}}=a_{\mu}\left[1+n\left(\omega_{\mu}, T\right)\right]^{-1 / 2},
\end{gathered}
$$

а матрица корреляционных функций примет вид

$$
\mathbf{J}(T)=\left(\begin{array}{cc}
0 & (1+n(\omega, T)) J(\omega) \\
n(\omega, T) J(\omega) & 0
\end{array}\right) .
$$

Собственные значения $\lambda$ и собственные векторы (правые $\overrightarrow{\mathbf{X}}$ и левые $\overrightarrow{\mathbf{Y}}$ ) матрицы $\mathbf{J}$ выглядят следующим образом:

$$
\begin{aligned}
& \lambda= \pm \sqrt{n(\omega, T)(1+n(\omega, T))} J(\omega), \\
& \vec{X}_{ \pm \lambda}=\left(\begin{array}{c}
\frac{(1+n(\omega, T))^{1 / 2}}{\sqrt{2} n(\omega, T)^{1 / 4}(1+n(\omega, T))^{1 / 4}} \\
\pm \frac{n(\omega, T)^{1 / 2}}{\sqrt{2} n(\omega, T)^{1 / 4}(1+n(\omega, T))^{1 / 4}}
\end{array}\right) \\
& \vec{Y}_{ \pm \lambda}^{+}=\left(\frac{n(\omega, T)^{1 / 2}}{\sqrt{2} n(\omega, T)^{1 / 4}(1+n(\omega, T))^{1 / 4}} \quad \pm \frac{(1+n(\omega, T))^{1 / 2}}{\sqrt{2} n(\omega, T)^{1 / 4}(1+n(\omega, T))^{1 / 4}}\right)
\end{aligned}
$$

Скоррелированные операторы $\mathbf{X}$ и $\mathbf{Y}$ имеют форму

$$
\begin{aligned}
\mathbf{X}_{\lambda} & =\frac{\sqrt{J(\omega)}}{2}\left[(1+n(\omega, T))^{1 / 2} b+n(\omega, T)^{1 / 2} b^{+}\right], \\
\mathbf{X}_{-\lambda} & =\frac{i \sqrt{J(\omega)}}{2}\left[(1+n(\omega, T))^{1 / 2} b-n(\omega, T)^{1 / 2} b^{+}\right], \\
\mathbf{Y}_{\lambda} & =\frac{\sqrt{J(\omega)}}{2}\left[n(\omega, T)^{1 / 2} b+(1+n(\omega, T))^{1 / 2} b^{+}\right], \\
\mathbf{Y}_{-\lambda} & =\frac{i \sqrt{J(\omega)}}{2}\left[n(\omega, T)^{1 / 2} b-(1+n(\omega, T))^{1 / 2} b^{+}\right] .
\end{aligned}
$$

Вместо них далее будем использовать набор операторов

$$
\begin{aligned}
& \mathbf{X}_{T}=\sqrt{1+n(\omega, T)} b+\sqrt{n(\omega, T)} b^{+}, \\
& \mathbf{Y}_{T}=\sqrt{1+n(\omega, T)} b-\sqrt{n(\omega, T)} b^{+},
\end{aligned}
$$




$$
\begin{aligned}
& \mathbf{X}_{T}^{+}=\sqrt{1+n(\omega, T)} b^{+}+\sqrt{n(\omega, T)} b, \\
& \mathbf{Y}_{T}^{+}=\sqrt{1+n(\omega, T)} b^{+}-\sqrt{n(\omega, T)} b
\end{aligned}
$$

который имеет аналогичный физический смысл, но более удобен, так как не содержит комплексных множителей. Набор операторов (23), (24) назовем тепловым расщеплением операторов рождения и уничтожения, так как они имеют схожие коммутационные соотношения (см. приложение В), при $T=0$ совпадают с $b$ и $b^{+}$, а при $T \rightarrow \infty$ стремятся к их полусумме и полуразности соответственно.

Уравнение (4) в операторной форме имеет вид [12]

$$
\begin{aligned}
\frac{\partial}{\partial t} \boldsymbol{\sigma}(t)= & -i\left[\mathbf{H}_{\mathrm{S}}, \boldsymbol{\sigma}(t)\right]+\frac{1}{2} J(\omega) \mathbf{X}_{T} \boldsymbol{\sigma}(t) \mathbf{X}_{T}^{+}+\frac{1}{2} J(\omega) \mathbf{Y}_{T} \boldsymbol{\sigma}(t) \mathbf{Y}_{T}^{+}- \\
& -\frac{1}{4} J(\omega)\left(\mathbf{X}_{T}^{+} \mathbf{X}_{T} \boldsymbol{\sigma}(t)+\mathbf{Y}_{T}^{+} \mathbf{Y}_{T} \boldsymbol{\sigma}(t)+\boldsymbol{\sigma}(t) \mathbf{X}_{T}^{+} \mathbf{X}_{T}+\boldsymbol{\sigma}(t) \mathbf{Y}_{T}^{+} \mathbf{Y}_{T}\right) .
\end{aligned}
$$

В этом уравнении нет явной зависимости от температуры: вся она содержится в операторах $\mathbf{X}_{T}$ и $\mathbf{Y}_{T}$.

Найдем производную среднего значения операторов $\mathbf{D}_{j}$ через $\mathbf{X}_{T}$ и $\mathbf{Y}_{T}$ :

$$
\begin{aligned}
\frac{\partial}{\partial t}\left\langle\mathbf{D}_{j}\right\rangle(t)= & \operatorname{tr}\left(\mathbf{D}_{j} \frac{\partial}{\partial t} \boldsymbol{\sigma}(t)\right)=-\operatorname{tr}\left(i \mathbf{D}_{j}\left[\mathbf{H}_{\mathrm{S}}, \boldsymbol{\sigma}(t)\right]\right)+ \\
& +\frac{1}{2} J(\omega) \operatorname{tr}\left(\mathbf{D}_{j} \mathbf{X}_{T} \boldsymbol{\sigma}(t) \mathbf{X}_{T}^{+}\right)+\frac{1}{2} J(\omega) \operatorname{tr}\left(\mathbf{D}_{j} \mathbf{Y}_{T} \boldsymbol{\sigma}(t) \mathbf{Y}_{T}^{+}\right)- \\
& -\frac{1}{4} J(\omega)\left(\operatorname{tr}\left(\mathbf{D}_{j} \mathbf{X}_{T}^{+} \mathbf{X}_{T} \boldsymbol{\sigma}(t)\right)+\operatorname{tr}\left(\mathbf{D}_{j} \mathbf{Y}_{T}^{+} \mathbf{Y}_{T} \boldsymbol{\sigma}(t)\right)+\right. \\
& \left.+\operatorname{tr}\left(\mathbf{D}_{j} \boldsymbol{\sigma}(t) \mathbf{X}_{T}^{+} \mathbf{X}_{T}\right)+\operatorname{tr}\left(\mathbf{D}_{j} \boldsymbol{\sigma}(t) \mathbf{Y}_{T}^{+} \mathbf{Y}_{T}\right)\right)= \\
= & \frac{1}{2} J(\omega) \operatorname{tr}\left(\mathbf{X}_{T}^{+} \mathbf{D}_{j} \mathbf{X}_{T} \boldsymbol{\sigma}(t)\right)+\frac{1}{2} J(\omega) \operatorname{tr}\left(\mathbf{Y}_{T}^{+} \mathbf{D}_{j} \mathbf{Y}_{T} \boldsymbol{\sigma}(t)\right)- \\
& -\frac{1}{4} J(\omega)\left[\operatorname{tr}\left(\mathbf{D}_{j} \mathbf{X}_{T}^{+} \mathbf{X}_{T} \boldsymbol{\sigma}(t)\right)+\operatorname{tr}\left(\mathbf{D}_{j} \mathbf{Y}_{T}^{+} \mathbf{Y}_{T} \boldsymbol{\sigma}(t)\right)+\right. \\
& \left.+\operatorname{tr}\left(\mathbf{X}_{T}^{+} \mathbf{X}_{T} \mathbf{D}_{j} \boldsymbol{\sigma}(t)\right)+\operatorname{tr}\left(\mathbf{Y}_{T}^{+} \mathbf{Y}_{T} \mathbf{D}_{j} \boldsymbol{\sigma}(t)\right)\right]=J(\omega) \operatorname{tr}\left(\mathbf{G}_{j} \boldsymbol{\sigma}(t)\right),
\end{aligned}
$$

где

$$
\begin{aligned}
\mathbf{G}_{j}= & \frac{1}{2} \mathbf{X}_{T}^{+} \mathbf{D}_{j} \mathbf{X}_{T}+\frac{1}{2} \mathbf{Y}_{T}^{+} \mathbf{D}_{j} \mathbf{Y}_{T}- \\
& -\frac{1}{4}\left(\mathbf{D}_{j} \mathbf{X}_{T}^{+} \mathbf{X}_{T}+\mathbf{D}_{j} \mathbf{Y}_{T}^{+} \mathbf{Y}_{T}+\mathbf{X}_{T}^{+} \mathbf{X}_{T} \mathbf{D}_{j}+\mathbf{Y}_{T}^{+} \mathbf{Y}_{T} \mathbf{D}_{j}\right) .
\end{aligned}
$$

Поскольку для произвольной диагональной матрицы плотности должно выполняться условие (10), то уравнение для операторов $\mathbf{D}_{j}$ может быть сформулировано так:

$$
\mathbf{G}_{j}=-j \mathbf{D}_{j}
$$

По индукции можно доказать, что решения этого уравнения удовлетворяют рекуррентной формуле

$$
\mathbf{D}_{j+1}=\mathbf{X}_{T}^{+} \mathbf{D}_{j} \mathbf{Y}_{T}+\mathbf{Y}_{T}^{+} \mathbf{D}_{j} \mathbf{X}_{T}+j^{2}\left[\mathbf{Y}_{T}, \mathbf{X}_{T}\right]^{2} \mathbf{D}_{j-1}
$$


с первыми членами

$$
\begin{gathered}
\mathbf{D}_{0}=1, \\
\frac{\mathbf{H}_{\mathrm{S}}-E_{\text {equil }}}{\hbar \omega}=\frac{1}{2}\left(b b^{+}+b^{+} b\right)-\left(n(\omega, T)+\frac{1}{2}\right)\left(b b^{+}-b^{+} b\right)= \\
=(1+n(\omega, T)) b^{+} b-n(\omega, T) b b^{+}=\frac{1}{2}\left(\mathbf{X}_{T}^{+} \mathbf{Y}_{T}+\mathbf{Y}_{T}^{+} \mathbf{X}_{T}\right), \\
\mathbf{D}_{1}=\mathbf{X}_{T}^{+} \mathbf{Y}_{T}+\mathbf{Y}_{T}^{+} \mathbf{X}_{T} .
\end{gathered}
$$

Если перейти обратно к операторам рождения и уничтожения, получим

$$
\mathbf{D}_{j+1}=(1+n(\omega, T)) b^{+} \mathbf{D}_{j} b+n(\omega, T) b \mathbf{D}_{j} b^{+}+4 j^{2} n(\omega, T)(1+n(\omega, T)) \mathbf{D}_{j-1} .
$$

В базисе собственных векторов гамильтониана системы соотношение (31) принимает вид

$$
d_{j+1, i}=(1+n(\omega, T)) i d_{j, i-1}+n(\omega, T)(i+1) d_{j, i+1}+4 j^{2} n(\omega, T)(1+n(\omega, T)) d_{j-1, i},
$$

где $d_{j i}-i$-е собственное число оператора $\mathbf{D}_{j}$ :

$$
\mathbf{D}_{j}=\sum_{i} d_{j i}|i\rangle\langle i| .
$$

Зная числа $d_{j i}$, можно получить набор матриц, соответствующих разложению диагональной части матрицы плотности на экспоненциально убывающие слагаемые:

$$
\begin{aligned}
\boldsymbol{\sigma}_{j}(T) & =\sum_{i}\left(\mathbf{D}(T)^{-1}\right)_{j, i}|i\rangle\langle i|, \\
\boldsymbol{\sigma}_{d}(t, T) & =\sum_{j} c_{j} \boldsymbol{\sigma}_{j}(T) e^{-j J(\omega) t},
\end{aligned}
$$

где $\mathbf{D}(T)^{-1}$ - матрица, обратная матрице $\mathbf{D}(T)$, составленной из элементов $d_{j i} ; c_{j}-$ коэффициенты, которые полностью определяются начальным состоянием системы. Таким образом, рекуррентная формула (31) дает возможность описать операторы экспоненциально убывающих наблюдаемых и определить через них динамику диагональной части редуцированной матрицы плотности, т.е. найти зависимость заселенностей колебательных уровней от времени.

\section{3. ЗАКЛЮЧЕНИЕ}

Мы рассмотрели задачу о диссипативной динамике гармонического осциллятора, линейно связанного с термостатом. Предложена методика представления временнь́х характеристик системы через операторы, соответствующие экспоненциально убывающим наблюдаемым. Доказано, что спектр констант затухания соответствующих наблюдаемых имеет вид

$$
k_{n}=n J(\omega) \text {, }
$$

где $n$ - неотрицательное целое число. 
Предложена новая форма записи уравнения для динамики редуцированной матрицы плотности гармонического осциллятора. В этой форме мы исходим из операторов $\mathbf{X}_{T}$ и $\mathbf{Y}_{T}$ (тепловое расщепление операторов рождения и уничтожения), которые получаются при диагонализации матрицы корреляционных функций. Через операторы $\mathbf{X}_{T}$ и $\mathbf{Y}_{T}$ получена рекуррентная формула (31) для операторов экспоненциально убывающих наблюдаемых.

Таким образом, мы показали, как, не решая систему уравнений Редфилда для диагональных элементов редуцированной матрицы плотности, с помощью уравнений (34) и (35) можно полностью описать динамику диссипации в гармоническом осцилляторе, линейно связанном с термостатом.

\section{ПРИЛОЖЕНИЕ А}

\section{Производная $n$-й степени энергии}

Рассмотрим дифференциальное уравнение для $n$-й степени энергии гармонического осциллятора с частотой $\omega$. Используя выражение для среднего значения через диагональные элементы матрицы плотности

$$
\left\langle\frac{E^{n}}{\hbar^{n} \omega^{n}}\right\rangle(t)=\sum_{i}\left(i+\frac{1}{2}\right)^{n} \sigma_{i i}(t)
$$

и применяя уравнение (4), находим

$$
\begin{aligned}
\frac{\partial}{\partial t}\left\langle\frac{E^{n}}{\hbar^{n} \omega^{n}}\right\rangle(t)= & \sum_{i=0}^{\infty}\left(i+\frac{1}{2}\right)^{n} \frac{\partial}{\partial t} \sigma_{i i}(t)= \\
= & \sum_{i=0}^{\infty}\left(i+\frac{1}{2}\right)^{n} i J(\omega) n(\omega)\left[\sigma_{i-1, i-1}(t)-\frac{1+n(\omega)}{n(\omega)} \sigma_{i i}(t)\right]- \\
& -\sum_{i=0}^{\infty}\left(i+\frac{1}{2}\right)^{n}(i+1) J(\omega) n(\omega)\left[\sigma_{i i}(t)-\frac{1+n(\omega)}{n(\omega)} \sigma_{i+1, i+1}(t)\right] .
\end{aligned}
$$

Сделаем замену индекса во второй сумме:

$$
\begin{aligned}
& \sum_{i=1}^{\infty}\left(i+\frac{1}{2}\right)^{n} i J(\omega) n(\omega)\left[\sigma_{i-1, i-1}(t)-\frac{1+n(\omega)}{n(\omega)} \sigma_{i i}(t)\right]- \\
& \quad-\sum_{i=1}^{\infty}\left(i-\frac{1}{2}\right)^{n} i J(\omega) n(\omega)\left[\sigma_{i-1, i-1}(t)-\frac{1+n(\omega)}{n(\omega)} \sigma_{i i}(t)\right]= \\
& =\sum_{i=1}^{\infty} i\left[\sum_{j=1}^{n} C_{j}^{n} \frac{i^{n-j}}{2^{j}}-\sum_{j=1}^{n} C_{j}^{n} \frac{(-1)^{j} i^{n-j}}{2^{j}}\right] J(\omega) n(\omega)\left[\sigma_{i-1, i-1}(t)-\frac{1+n(\omega)}{n(\omega)} \sigma_{i i}(t)\right]= \\
& =\sum_{i=1}^{\infty}\left[n i^{n}+\sum_{j=3}^{n} C_{j}^{n} \frac{i^{n-j}}{2^{j}}-\sum_{j=3}^{n} C_{j}^{n} \frac{(-1)^{j} i^{n-j}}{2^{j}}\right] J(\omega) n(\omega)\left[\sigma_{i-1, i-1}(t)-\frac{1+n(\omega)}{n(\omega)} \sigma_{i i}(t)\right]= \\
& =\sum_{i=1}^{\infty} J(\omega)\left[n i^{n}+\sum_{j=1}^{n / 2} C_{2 j+1}^{n} \frac{i^{n-(2 j+1)}}{2^{2 j}}\right]\left[n(\omega) \sigma_{i-1, i-1}(t)-(1+n(\omega)) \sigma_{i i}(t)\right]=
\end{aligned}
$$




$$
\begin{aligned}
= & \sum_{i=0}^{\infty} J(\omega)\left[n(i+1)^{n}+\sum_{j=1}^{n / 2} C_{2 j+1}^{n} \frac{(i+1)^{n-(2 j+1)}}{2^{2 j}}\right] n(\omega) \sigma_{i i}(t)- \\
& -\sum_{i=1}^{\infty} J(\omega)\left[n i^{n}+\sum_{j=1}^{n / 2} C_{2 j+1}^{n} \frac{i^{n-(2 j+1)}}{2^{2 j}}\right](1+n(\omega)) \sigma_{i i}(t) .
\end{aligned}
$$

Перейдем к разложению по степеням $i+1 / 2$. Коэффициент при $n$-й степени будет совпадать с коэффициентом при $i^{n}$ в исходном уравнении:

$$
\begin{aligned}
& \frac{\partial}{\partial t}\left\langle\frac{E^{n}}{\hbar^{n} \omega^{n}}\right\rangle(t)= \\
& \quad=\sum_{i=0}^{\infty} J(\omega)\left[n\left(i+\frac{1}{2}\right)^{n} n(\omega)-n\left(i+\frac{1}{2}\right)^{n}(1+n(\omega))+\sum_{j=1}^{n} \alpha_{n j}\left(i+\frac{1}{2}\right)^{n-j}\right] \sigma_{i i}(t)= \\
& \left.\quad=-n J(\omega) \sum_{i=0}^{\infty}\left(i+\frac{1}{2}\right)^{n} \sigma_{i i}(t)+\text { (сумма более низких степеней }\right) \times\left(i+\frac{1}{2}\right), \quad \text { (П.2) }
\end{aligned}
$$

где точная форма коэффициентов $\alpha_{n j}$ не имеет значения. Тем самым соотношение (12) доказано.

\section{ПРИЛОЖКЕНИЕ Б}

\section{Построение матрицы корреляционных функций}

Построим набор ортонормированных операторов термостата. Для этого рассмотрим равновесную матрицу плотности термостата. Так как его гамильтониан может быть разбит на сумму коммутирующих операторов, соответствующих разным модам, то матрица плотности может быть разложена в произведение:

$$
\boldsymbol{\sigma}_{\mathrm{B}}(T)=\frac{1}{Z(T)} e^{-\hbar \sum_{\mu} \omega_{\mu}\left(a_{\mu}^{+} a_{\mu}+1 / 2\right) /(k T)}=\prod_{\mu} \frac{e^{-\hbar \omega_{\mu}\left(a_{\mu}^{+} a_{\mu}+1 / 2\right) /(k T)}}{Z_{\mu}(T)},
$$

где $Z_{\mu}(T)$ - сумма по состояниям, соответствующая $\mu$-й моде:

$$
\begin{aligned}
Z_{\mu}(T) & =\sum_{i=0}^{\infty} e^{-\hbar \omega_{\mu}(i+1 / 2) /(k T)}=e^{-\hbar \omega_{\mu} /(2 k T)} \sum_{i=0}^{\infty} e^{-i \hbar \omega_{\mu} /(k T)}= \\
& =e^{-\hbar \omega_{\mu} /(2 k T)}\left[1-e^{-\hbar \omega_{\mu} /(k T)}\right]^{-1} .
\end{aligned}
$$

Набор операторов $\left\{a_{\mu}, a_{\mu}^{+}\right\}$является ортогональным, так как $\operatorname{tr}_{\mathrm{B}}\left[a_{\mu} \boldsymbol{\sigma}_{\mathrm{B}}(T) a_{\nu}^{+}\right]=$ $\operatorname{tr}_{\mathrm{B}}\left[a_{\nu}^{+} a_{\mu} \boldsymbol{\sigma}_{\mathrm{B}}(T)\right]$, а оператор $a_{\nu}^{+} a_{\mu}$ содержит диагональные члены только при $\mu=\nu$, a $\operatorname{tr}_{\mathrm{B}}\left[a_{\mu} \boldsymbol{\sigma}_{\mathrm{B}}(T) a_{\nu}\right]=0$ - при любых $\mu$ и $\nu$. Приведем его к ортонормированному виду:

$$
\begin{aligned}
& \operatorname{tr}_{\mathrm{B}}\left[a_{\mu} \boldsymbol{\sigma}_{\mathrm{B}}(T) a_{\mu}^{+}\right]=\operatorname{tr}_{\mathrm{B}}\left[a_{\mu} \prod_{\mu} \frac{e^{-\hbar \omega_{\mu}\left(a_{\mu}^{+} a_{\mu}+1 / 2\right) /(k T)}}{Z_{\mu}(T)} a_{\mu}^{+}\right]= \\
& \quad=\operatorname{tr}_{\mathrm{B}}\left[a_{\mu} \frac{e^{-\hbar \omega_{\mu}\left(a_{\mu}^{+} a_{\mu}+1 / 2\right) /(k T)}}{Z_{\mu}(T)} a_{\mu}^{+}\right]=\frac{1}{Z_{\mu}(T)} \operatorname{tr}_{\mu}\left[a_{\mu} e^{-\hbar \omega_{\mu}\left(a_{\mu}^{+} a_{\mu}+1 / 2\right) /(k T)} a_{\mu}^{+}\right]=
\end{aligned}
$$




$$
\begin{aligned}
& =\frac{1-e^{-\hbar \omega_{\mu} /(k T)}}{e^{-\hbar \omega_{\mu} /(2 k T)}} \operatorname{tr}_{\mu}\left[\sum_{i=0}^{\infty} e^{-\hbar \omega_{\mu}(i+1 / 2) /(k T)} a_{\mu}\left|i_{\mu}\right\rangle\left\langle i_{\mu}\right| a_{\mu}^{+}\right]= \\
& =\left[1-e^{-\hbar \omega_{\mu} /(k T)}\right] \operatorname{tr}_{\mu}\left[\sum_{i=0}^{\infty} i e^{-\hbar \omega_{\mu} i /(k T)}\left|i_{\mu}-1\right\rangle\left\langle i_{\mu}-1\right|\right]= \\
& =\left[1-e^{-\hbar \omega_{\mu} /(k T)}\right] \sum_{i=0}^{\infty} i e^{-\hbar \omega_{\mu} i /(k T)}= \\
& =\left[1-e^{-\hbar \omega_{\mu} /(k T)}\right] \frac{e^{-\hbar \omega_{\mu} /(k T)}}{\left[1-e^{-\hbar \omega_{\mu} /(k T)}\right]^{2}}=\frac{e^{-\hbar \omega_{\mu} /(k T)}}{1-e^{-\hbar \omega_{\mu} /(k T)}},
\end{aligned}
$$

где $\operatorname{tr}_{\mu}$ обозначает процедуру взятия следа не по всем координатам термостата, а только по $\mu$-й колебательной моде.

Аналогично можно показать, что

$$
\operatorname{tr}_{\mathrm{B}}\left[a_{\mu}^{+} \boldsymbol{\sigma}_{\mathrm{B}}(T) a_{\mu}\right]=\frac{1}{1-e^{-\hbar \omega_{\mu} /(k T)}} .
$$

Следовательно, ортонормированный набор операторов термостата примет вид

$$
\begin{aligned}
q_{-\mu} & =a_{\mu}^{+} \sqrt{\frac{1-e^{-\hbar \omega_{\mu} /(k T)}}{e^{-\hbar \omega_{\mu} /(k T)}}}, \\
q_{\mu} & =a_{\mu} \sqrt{\frac{1}{1-e^{-\hbar \omega_{\mu} /(k T)}}} .
\end{aligned}
$$

\section{Свойства операторов $\mathbf{X}_{T}, \mathbf{Y}_{T}$}

Одним из основных свойств операторов $\mathbf{X}_{T}$ и $\mathbf{Y}_{T}$ является их коммутационное соотношение с эрмитово сопряженным оператором:

$$
\left[\mathbf{X}_{T}, \mathbf{X}_{T}^{+}\right]=\left[\mathbf{Y}_{T}, \mathbf{Y}_{T}^{+}\right]=1
$$

Это соотношение позволяет рассматривать операторы $\mathbf{X}_{T}$ и $\mathbf{Y}_{T}$ как операторы уничтожения, а $\mathbf{X}_{T}^{+}$и $\mathbf{Y}_{T}^{+}$- как соответствующие им операторы рождения.

Операторы $\mathbf{X}_{T}$ и $\mathbf{Y}_{T}$ удовлетворяют коммутационным соотношениям

$$
\left[\mathbf{Y}_{T}, \mathbf{X}_{T}\right]=\left[\mathbf{X}_{T}^{+}, \mathbf{Y}_{T}^{+}\right]=2 \sqrt{n(\omega, T)(1+n(\omega, T))} .
$$

Также имеет место третья пара коммутационных соотношений:

$$
\left[\mathbf{Y}_{T}, \mathbf{X}_{T}^{+}\right]=\left[\mathbf{X}_{T}, \mathbf{Y}_{T}^{+}\right]=2 n(\omega, T)+1
$$

Операторы $\mathbf{X}_{T}$ и $\mathbf{Y}_{T}$ линейно зависимы:

$$
\begin{gathered}
Y_{T}=(1+2 n(\omega, T)) X_{T}-2 \sqrt{n(\omega, T)(1+n(\omega, T))} X_{T}^{+}, \\
Y_{T}^{+}=(1+2 n(\omega, T)) X_{T}^{+}-2 \sqrt{n(\omega, T)(1+n(\omega, T))} X_{T} .
\end{gathered}
$$


Благодарности. Работа выполнена при финансовой поддержке РФФИ (грант № 09-03-00889) и Федерального агентства по образованию, программа "Научные и научно-педагогические кадры инновационной России на 2009-2013 годы" (контракты П2100, П2280).

\section{Список литературы}

[1] A. G. Redfield, Adv. Magn. Res., 1 (1965), 1-32.

[2] V. Sundström, Progr. Quantum Electron., 24:5 (2000), 187-238.

[3] R. S. Knox, J. Photochem. Photobiol. B, 49:2-3 (1999), 81-88.

[4] C. E. Crespo-Hernández, B. Cohen, P. M. Hare, B. Kohler, Chem. Rev., 104:4 (2004), 1977-2020.

[5] S. K. Pal, A. H. Zewail, Chem. Rev., 104:4 (2004), 2099-2124.

[6] X. Hu, T. Ritz, A. Damjanovic, K. Schulten, J. Phys. Chem. B, 101:19 (1997), 3854-3871.

[7] D. Egorova, A. Kühl, W. Domcke, Chem. Phys., 268:1-3 (2001), 105-120.

[8] D. Egorova, W. Domcke, J. Photochem. Photobiol. A, 166:1-3 (2004), 19-31.

[9] A. Kühl, W. Domcke, J. Chem. Phys., 116:1 (2002), 263-274.

[10] И. О. Глебов, В. В. Еремин, Журн. физ. химии, 82:4 (2008), 684-689.

[11] V. I. Novoderezhkin, A. G. Yakovlev, R. van Grondelle, V. A. Shuvalov, J. Phys. Chem. B, 108(22) (2004), 7445-7457.

[12] И. О. Глебов, В. В. Еремин, ТМФ, 161:1 (2009), 83-94.

[13] В. В. Еремин, И. О. Глебов, ТМФ, 153:1 (2007), 130-144.

[14] К. Блум, Теория матрицы плотности и ее приложения, Мир, М., 1983.

[15] U. Weiss, Quantum Dissipative Systems, 2nd ed., Ser. Modern Condensed Matter Phys., 10, World Sci., Singapore, 1999. 